\title{
Magnetoelectric point scattering theory for metamaterial scatterers
}

\author{
Ivana Sersic, ${ }^{*}$ Christelle Tuambilangana, Tobias Kampfrath, ${ }^{\dagger}$ and A. Femius Koenderink \\ Center for Nanophotonics, FOM Institute for Atomic and Molecular Physics (AMOLF), Science Park 104, \\ 1098 XG Amsterdam, The Netherlands \\ (Received 16 December 2010; published 3 June 2011)
}

\begin{abstract}
We present a fully analytical point scattering model that can be applied to arbitrary anisotropic magnetoelectric dipole scatterers, including split ring resonators (SRRs) and chiral and anisotropic plasmonic scatterers. We have taken proper account of reciprocity and radiation damping for electric and magnetic scatterers with any general polarizability tensor, including magnetoelectric interactions. Our theory sheds new light on the magnitude of cross sections for scattering and extinction, and, for instance, on the emergence of structural chirality in the optical response of geometrically nonchiral scatterers such as SRRs and SRR clusters. Specifically, we predict which observables in scattering experiments allow to fully quantify all components of the polarizability tensor of SRR's, including their off-diagonal magnetoelectric response. Finally, we show that our model describes well the extinction of stereodimers of split rings, while providing a completely new interpretation of the coupling mechanisms underlying recent experiments.
\end{abstract}

DOI: 10.1103/PhysRevB.83.245102

PACS number(s): 42.70.-a, 42.25.-p, 78.20.Bh

\section{INTRODUCTION}

Research in the field of metamaterials is driven by the possibility to control the properties of light on the nanoscale by using coupled resonant nanoscatterers to create optical materials with very unusual effective-medium parameters. Engineering arbitrary values for the effective permittivity $\epsilon$ and permeability $\mu$ would allow new forms of light control based on achieving negative index materials, ${ }^{1-3}$ or transformation optics media ${ }^{4}$ that arbitrarily reroute light through space. To reach such control over $\epsilon$ and $\mu$, many metamaterial building nanoblocks have previously been identified as having an electric and magnetic response to incident light, including split ring resonators (SRR's), ${ }^{5-10}$ rod pairs, ${ }^{11}$ cut-wire pairs, ${ }^{12}$ fishnet structures, ${ }^{13-15}$ and coaxial waveguides. ${ }^{16}$ In many instances, the nanoscatterers are not only interesting as building blocks in subwavelength lattices of designed $\epsilon$ and $\mu$. The building blocks are in fact very strong scatterers with large cross sections, ${ }^{17-20}$ comparable to the large cross sections of plasmonic structures. Therefore, metamaterial building blocks are excellently suited to construct magnetic antennas, array waveguides, and gratings in which electric and magnetic dipoles couple and form cooperative excitations, in analogy to the functionality imparted by plasmon hybridization. ${ }^{21}$ Experiments outside the domain of effective media have appeared only recently. These include experiments by Husnik et $a l .{ }^{17}$ and Banzer et al. ${ }^{22}$ that quantify the extinction cross section of single split rings under differently polarized illumination, experiments where split ring resonators act as near-field probes, ${ }^{23}$ as well as a suite of experiments on coupled systems. These experiments include extinction measurements on split ring dimers ${ }^{24}$ that point to resonance hybridization, as well as reports of magnetization waves ${ }^{25}$ and structural and geometrical chirality in arrays, as is evident in, e.g., massive circular dichroism, ${ }^{26-33}$ and chiral effects in split ring stereodimers studied by Liu et al. ${ }^{34}$

To understand the light-metamatter interaction in systems of strongly coupled magnetoelectric scatterers, it is important to understand how individual metamaterial building blocks are excited and how they scatter. So far, explanations of the observed phenomena have mainly rested on two pillars. On the one hand, data are compared to brute force finitedifference time-domain (FDTD) simulations of Maxwell's equations, usually showing good correspondence. $5,6,8,17,18,26,27$ The FDTD method is essentially equivalent to performing a rigorous numerical experiment that still requires further interpretation to understand how split rings scatter or hybridize in coupled systems. There is general consensus that to lowest order, metamaterial interactions in lattices of scatterers like SRR's must be described by magnetoelectric point-dipole interactions. Hence, simple models with dipolar coupling terms are the second main interpretative tool to predict, e.g., frequency shifts due to electric and magnetic dipole-dipole interactions in lattices and oligomers. These models either take the form of dipole models in which electrostatic and magnetostatic polarizabilities are coupled to predict, e.g., $\epsilon$ and $\mu$, and the existence of coupled excitations like magnetoinductive waves, ${ }^{35-41}$ or they take the form of Lagrangian equations of motion for current oscillation in coupled $L C$ circuits. ${ }^{9,24,34,42}$ To rationalize this $L C$ circuit intuition, several authors have analyzed current distributions obtained by FDTD simulations to retrieve the microscopic parameters (i.e., the polarizability) underlying such a dipolar interaction model, and to estimate multipolar corrections. ${ }^{18-20,43-46}$

While there is general consensus that to lowest order, metamaterial interactions must essentially be magnetoelectric point dipole interactions, we note that the dipolar circuit models that are adequate to explain many basic features of subwavelength clusters and metamaterial arrays have significant limitations when applied to strong scatterers and larger clusters. This is a consequence of the fact that electric circuit theories lack the velocity of light $c$ as a parameter, by assuming $c=\infty(k=0) .{ }^{9,24,34,42}$ Such theories will be referred to in this work as "quasistatic" or "electrostatic" and "magnetostatic." Their validity ends when retardation or interference become important, quantitative cross sections are required, or super- and subradiant radiative damping plays a role. A fair comparison of experiments with intuitive dipole requires a fully electrodynamic theory that (i) contains finite $c$, (ii) satisfies energy conservation in the form of an appropriate 
optical theorem, and (iii) can be used for dipoles with tensorial electric, magnetic, and magnetoelectric response. Indeed, actual metamaterial scatterers are very strongly bi-anisotropic, with large magnetoelectric cross coupling whereby magnetic fields induce strong electric dipoles and vice versa. We note that the theories existing so far can be divided in two classes. On the one hand, $L C$ models and electrostatic and magnetostatic polarizability approaches ${ }^{9,24,34-42}$ correctly deal with (iii), but not with (i) and (ii). On the other hand, textbook electric point dipole scattering theories exist that correctly deal with (i) and (ii), but not with magnetoelectric effects (iii). Electric point dipole scattering theory is well known as a very effective means to describe random media, extraordinary transmission, and plasmon particle arrays, ${ }^{47-49}$ and it has been applied to metamaterial problems, but only in cases without magnetoelectric effects. ${ }^{50,51}$ In this paper, we derive an electrodynamic theory for general magnetoelectric scatterers that satisfies all three requirements. Essential ingredients for a consistent theory are generalized Onsager constraints $41,52,53$ for the electrodynamic polarizability, and a magnetoelectric optical theorem first derived by Belov. ${ }^{54}$ While our theory sheds no light on the microscopic origin of the polarizability, ${ }^{55}$ a unique contribution is that we show how any quasistatic model $1^{9,24,34-42}$ can be converted into an electrodynamically consistent scattering theory by a new tensorial method of radiation damping addition. Furthermore, we predict how extinction measurements and measurements of radiation patterns (i.e., differential scattering cross section) can be used to quantify the polarizability tensor.

The paper is structured in the following way: In Sec. II, we review in detail the general theory, taking into full account reciprocity, the optical theorem, and radiation damping. In Sec. III, we apply this theory to set up the polarizability of the archetypical metamaterial building block, a single SRR. In Sec. IV, we show which set of experiments can be used to retrieve the tensor polarizability $\boldsymbol{\alpha}$. We find that magnetoelectric coupling directly implies circular dichroism in the extinction of single split rings, evidencing the utility of our theory to describe structural chirality. ${ }^{26-33} \mathrm{We}$ show in Sec. V that the theory can be simply applied to obtain quantitative scattering spectra of coupled systems. By way of example, we examine the case of two coupled resonators in the stereodimer configuration reported by Liu et $a .^{34}$

\section{MAGNETOELECTRIC POINT SCATTERER}

\section{A. Dynamic polarizability}

A paradigm in scattering theory is the point dipole scatterer $^{4-49,56,57}$ to model scattering by very small but strongly scattering particles. In such a theory, each scatterer is approximated as an electric dipole with an electric dipole moment $\boldsymbol{p}=\alpha_{E E} \boldsymbol{E}$ that is proportional to the driving electric field $\boldsymbol{E}$. The proportionality constant is the polarizability $\alpha_{E E}$. Generally, incident fields $\boldsymbol{E}$ and $\boldsymbol{H}$ induce a (complex) current distribution in an arbitrary scatterer. It is the express point of this paper to assess what the scattering properties are of subwavelength scatterers with strong electric and magnetic dipole moments, as this represents the physics expected of metamaterial building blocks. ${ }^{9,24,34,42,58}$ Therefore, we retain only electric and magnetic dipole terms, neglecting higherorder multipoles. We derive a generalized point scattering theory for metamaterials that includes a magnetic dipole moment $\boldsymbol{m}$ on an equal footing with the electric dipole moment $\boldsymbol{p}$. By definition, the electric and magnetic fields due to an induced $\boldsymbol{p}$ and $\boldsymbol{m}$ are equal to

$$
\left(\begin{array}{c}
\boldsymbol{E}_{\text {out }} \\
\boldsymbol{H}_{\text {out }}
\end{array}\right)=\boldsymbol{G}^{0}\left(\boldsymbol{r}, \boldsymbol{r}^{\prime}\right)\left(\begin{array}{c}
\boldsymbol{p} \\
\boldsymbol{m}
\end{array}\right),
$$

with a dyadic Green tensor $\boldsymbol{G}^{0}$ that describes the field at position $\boldsymbol{r}=(x, y, z)$ due to a dipole at $\boldsymbol{r}^{\prime}=\left(x^{\prime}, y^{\prime}, z^{\prime}\right)$. Throughout this paper, we suppress harmonic time dependence $e^{-i \omega t}$. We use a rationalized unit system that significantly simplifies all equations and is fully explained in the Appendix. The $6 \times 6$ Green dyadic of free space can be divided into four $3 \times 3$ blocks,

$$
\boldsymbol{G}^{0}\left(\boldsymbol{r}, \boldsymbol{r}^{\prime}\right)=\left(\begin{array}{ll}
\boldsymbol{G}_{E E}^{0}\left(\boldsymbol{r}, \boldsymbol{r}^{\prime}\right) & \boldsymbol{G}_{E H}^{0}\left(\boldsymbol{r}, \boldsymbol{r}^{\prime}\right) \\
\boldsymbol{G}_{H E}^{0}\left(\boldsymbol{r}, \boldsymbol{r}^{\prime}\right) & \boldsymbol{G}_{H H}^{0}\left(\boldsymbol{r}, \boldsymbol{r}^{\prime}\right)
\end{array}\right) .
$$

The $3 \times 3$ diagonals correspond to the familiar known electricfield Green dyadic ${ }^{48,49}$ and magnetic-field Green dyadic of free space, which in our unit system (see the Appendix) both equal

$$
\boldsymbol{G}_{E E}^{0}\left(\boldsymbol{r}, \boldsymbol{r}^{\prime}\right)=\boldsymbol{G}_{H H}^{0}\left(\boldsymbol{r}, \boldsymbol{r}^{\prime}\right)=\left(\mathbb{I} k^{2}+\nabla \nabla\right) \frac{e^{i k\left|\boldsymbol{r}-\boldsymbol{r}^{\prime}\right|}}{\left|\boldsymbol{r}-\boldsymbol{r}^{\prime}\right|} .
$$

The off-diagonal blocks correspond to the mixed dyadics that specify the electric field at $\boldsymbol{r}$ due to a magnetic dipole at $\boldsymbol{r}^{\prime}$, and the magnetic field at $\boldsymbol{r}$ due to an electric dipole at $\boldsymbol{r}^{\prime}$, respectively. Explicitly,

$$
\begin{aligned}
\boldsymbol{G}_{E H}^{0}\left(\boldsymbol{r}, \boldsymbol{r}^{\prime}\right) & =-\boldsymbol{G}_{H E}^{0}\left(\boldsymbol{r}, \boldsymbol{r}^{\prime}\right) \\
& =i k\left(\begin{array}{ccc}
0 & \partial_{z} & -\partial_{y} \\
-\partial_{z} & 0 & \partial_{x} \\
\partial_{y} & -\partial_{x} & 0
\end{array}\right) \frac{e^{i k\left|\boldsymbol{r}-\boldsymbol{r}^{\prime}\right|}}{\left|\boldsymbol{r}-\boldsymbol{r}^{\prime}\right|}
\end{aligned}
$$

The central starting point in this work is that we assume that $\boldsymbol{p}$ and $\boldsymbol{m}$ can be induced by both the external electric and magnetic fields $\boldsymbol{E}$ and $\boldsymbol{H}$ according to the most general linear response,

$$
\left(\begin{array}{c}
\boldsymbol{p} \\
\boldsymbol{m}
\end{array}\right)=\alpha\left(\begin{array}{c}
\boldsymbol{E}_{\mathrm{in}} \\
\boldsymbol{H}_{\mathrm{in}}
\end{array}\right) .
$$

In Eq. (5), $\alpha$ is a $6 \times 6$ polarizability tensor, which consists of four $3 \times 3$ blocks, each of which describes part of the dipole response to the electric or magnetic component of the incident light,

$$
\boldsymbol{\alpha}=\left(\begin{array}{cc}
\boldsymbol{\alpha}_{E E} & \boldsymbol{\alpha}_{E H} \\
\boldsymbol{\alpha}_{H E} & \boldsymbol{\alpha}_{H H}
\end{array}\right)
$$

This form and interpretation of the $6 \times 6$ polarizability in Eq. (6) is very well known in the field of bi-isotropic and bianisotropic media. In this field, many authors have analyzed the electrostatic and magnetostatic polarizability of $\Omega$ particles, split ring resonators, bi-(an)isotropic spheres in the Rayleigh limit, and chiral scatterers such as wire helices. ${ }^{35-39,41,53}$ In 
addition to the purely electric polarizability $\boldsymbol{\alpha}_{E E}$ known from plasmonics, ${ }^{59}$ the tensor also contains $\boldsymbol{\alpha}_{H H}$, which quantifiesthe magnetic dipole induced purely by a driving magnetic field. Finally, the off-diagonal blocks represent magnetoelectric coupling. The lower diagonal $\boldsymbol{\alpha}_{H E}$ quantifies the magnetic dipole induced by an incident electric field, and $\boldsymbol{\alpha}_{E H}$ quantifies the electric dipole induced by an incident magnetic field. Such magnetoelectric coupling is well known to occur in the constitutive tensors of metamaterials. ${ }^{7,60}$ However, the relative strength of magnetoelectric coupling in the polarizability, i.e., $\boldsymbol{\alpha}_{E H}$ and $\boldsymbol{\alpha}_{H E}$, has not been experimentally quantified for the archetypical building blocks, such as split ring resonators.

An important distinction with earlier analyses of the $6 \times 6$ polarizability tensor in quasistatic models $\mathrm{s}^{35-39,41,53}$ is that in any scattering theory, $\boldsymbol{\alpha}$ is constrained by reciprocity and energy conservation. As we discuss below, energy conservation requires that the polarizability $\boldsymbol{\alpha}$ must depend on frequency $\omega$ and the velocity of light $c$. Hence, the polarizability that we discuss in this work is not a static polarizability, but is known as dynamic polarizability. ${ }^{47,48}$ This dynamic polarizability is equivalent to the single scatterer $t$-matrix. ${ }^{48}$

\section{B. Onsager relation for dynamic polarizability}

There are several constraints on $\boldsymbol{\alpha}$. In addition to any symmetry of the scatterer itself that may impose zeros in the polarizability tensor, these constraints are due to reciprocity and to energy conservation. We start by examining the constraints imposed by reciprocity. It is well known from the field of bi-anisotropic materials ${ }^{53}$ that reciprocity imposes so-called Onsager constraints on the most general constitutive tensors relating $(\boldsymbol{D}, \boldsymbol{B})$ to $(\boldsymbol{E}, \boldsymbol{H})$. Several textbooks ${ }^{41,53}$ and García-García et al. ${ }^{58}$ proposed that such Onsager constraints carry over directly to static polarizabilities. Reference 41 (Chap. 7.1) lists Onsager relations for the static polarizability in the following form:

$$
\boldsymbol{\alpha}_{E E}=\boldsymbol{\alpha}_{E E}^{T}, \quad \boldsymbol{\alpha}_{H H}=\boldsymbol{\alpha}_{H H}^{T}, \quad \text { and } \quad \boldsymbol{\alpha}_{E H}=-\boldsymbol{\alpha}_{H E}^{T} .
$$

These textbooks ${ }^{41,53}$ derive the Onsager relations rigorously for constitutive tensors $(\epsilon, \mu$, and cross terms) by solving the wave equation in homogeneous, bi-anisotropic space. To the best of our knowledge, the quoted Onsager relations for static polarizabilities are based on the assumption that Onsager relations derived for effective-medium tensors must hold also for microscopic constituent polarizabilities, as we could not find any independent proof in the literature. Here we rigorously derive the Onsager relations Eq. (7) for the dynamic polarizability of electrodynamic magnetoelectric point scatterers, and thereby show that the general point dipoles proposed in this work can indeed be used as microscopic building blocks for an exact scattering theory that describes the formation of bi-anisotropic media from dense lattices of scatterers in the effective-medium limit. Since the point scattering building blocks fulfill the Onsager constraints, they are indeed the natural building blocks to derive effective media constitutive tensors by homogenization that also satisfy the Onsager relations.

In this work, we focus solely on scatterers made from reciprocal materials (typically gold and silver), as is commonly true for the metallic scatterers that constitute metamaterials. Since the materials that compose our scatterers are assumed to satisfy reciprocity microscopically, the polarizability tensor must also lead to a scattering theory that satisfies reciprocity. To derive reciprocity constraints on $\boldsymbol{\alpha}$, it is sufficient to examine the Green function in the presence of just one point scatterer at the origin. This Green function that quantifies the field at $\boldsymbol{r}_{2}$ due to a source at $\boldsymbol{r}_{1}$ in the presence of a single scatterer at $\boldsymbol{r}_{s}$ can be written as $47,48,61$

$$
\boldsymbol{G}\left(\boldsymbol{r}_{2}, \boldsymbol{r}_{1}\right)=\boldsymbol{G}^{0}\left(\boldsymbol{r}_{2}, \boldsymbol{r}_{1}\right)+\boldsymbol{G}^{0}\left(\boldsymbol{r}_{2}, \boldsymbol{r}_{s}\right) \boldsymbol{\alpha} \boldsymbol{G}^{0}\left(\boldsymbol{r}_{s}, \boldsymbol{r}_{1}\right) .
$$

Reciprocity requires for any Green function $\boldsymbol{G}$ (similarly split in four blocks) that

$$
\begin{gathered}
\left(\begin{array}{ll}
\boldsymbol{G}_{E E}\left(\boldsymbol{r}_{2}, \boldsymbol{r}_{1}\right) & \boldsymbol{G}_{E H}\left(\boldsymbol{r}_{2}, \boldsymbol{r}_{1}\right) \\
\boldsymbol{G}_{H E}\left(\boldsymbol{r}_{2}, \boldsymbol{r}_{1}\right) & \boldsymbol{G}_{H H}\left(\boldsymbol{r}_{2}, \boldsymbol{r}_{1}\right)
\end{array}\right) \\
=\left(\begin{array}{cc}
\boldsymbol{G}_{E E}\left(\boldsymbol{r}_{1}, \boldsymbol{r}_{2}\right) & -\boldsymbol{G}_{E H}\left(\boldsymbol{r}_{1}, \boldsymbol{r}_{2}\right) \\
-\boldsymbol{G}_{H E}\left(\boldsymbol{r}_{1}, \boldsymbol{r}_{2}\right) & \boldsymbol{G}_{H H}\left(\boldsymbol{r}_{1}, \boldsymbol{r}_{2}\right)
\end{array}\right)^{T},
\end{gathered}
$$

which is equivalent to noting that swapping the source and the detector leaves the detected field unchanged, up to a change in sign. An extra minus occurs for the off-diagonal terms, i.e., when swapping a magnetic (electric) detector with an electric (magnetic) source. It is easy to verify that Eq. (9) is indeed satisfied by the free-space Green function $\boldsymbol{G}^{0}$.

Using this fact, we evaluate Eq. (9) for the Green function in Eq. (8) to find if reciprocity constrains $\boldsymbol{\alpha}$. Since reciprocity is clearly satisfied for the first term in Eq. (8), we now focus on verifying if:

$$
\boldsymbol{G}^{0}\left(\boldsymbol{r}_{2}, \boldsymbol{r}_{s}\right) \boldsymbol{\alpha} \boldsymbol{G}^{0}\left(\boldsymbol{r}_{s}, \boldsymbol{r}_{1}\right)=\left[\boldsymbol{G}^{0}\left(\boldsymbol{r}_{1}, \boldsymbol{r}_{s}\right) \boldsymbol{\alpha} \boldsymbol{G}^{0}\left(\boldsymbol{r}_{s}, \boldsymbol{r}_{2}\right)\right]^{\mathrm{T}-},
$$

Where [.] ${ }^{\mathrm{T}-}$ means transpose, and reverse sign of off-diagonal blocks, as in Eq. (9). Expanding the matrix products in Eq. (9) while making use of the reciprocity of the free Green function results in the Onsager relations for the dynamic polarizability that are listed in Eq. (7). These relations are identical in form to the Onsager relations for constitutive tensors, ${ }^{53}$ but are now derived on very different grounds. Whereas for bi-anisotropic media, Onsager relations on constitutive tensors are derived by solving for the Green function of homogeneous bi-anisotropic space, our new proof for Onsager relations that constrain dynamic polarizabilities only uses reciprocity of the Green function of vacuum.

\section{Tensorial magnetoelectric optical theorem}

Energy conservation imposes an "optical theorem" that constrains the dynamic polarizability such that (in the absence of material absorption) extinction equals scattering. The optical theorem for scalar electric dipole scatterers has been amply discussed in the literature, for instance in the work by Sipe and Van Kranendonk ${ }^{62}$ on resonant dielectrics, as well as in various references dealing with multiple scattering theory of random media and plasmonics. ${ }^{47-49}$ This same scalar optical theorem has also been applied in the context of metamaterials to scatterers with a magnetic response $\mathrm{e}^{50,51}$ that is completely uncoupled from the electric response. Extension of the optical theorem to $6 \times 6$ tensorial scatterers, i.e., including magnetoelectric cross coupling, is not immediately trivial as one needs to reevaluate the definition of extinction, and of 
the amount of power radiated into the far field. Such an extension to the general case is reported by Belov et al. in Ref. 54.

For completeness, we summarize the derivation of this optical theorem reported by Belov et al. ${ }^{54}$ As in the scalar electric case, ${ }^{47-49,62}$ the optical theorem is based on equating extinction to scattering. Here, extinction corresponds to the amount of work done per unit cycle by the incident field $\boldsymbol{E}_{\text {in }}$ and $\boldsymbol{H}_{\text {in }}$ to drive $\boldsymbol{p}$ and $\boldsymbol{m}$, which is equal to $W=$ $\left\langle\left\langle\operatorname{Re} \boldsymbol{E}_{\text {in }} \cdot \operatorname{Re} \frac{d \boldsymbol{p}}{d t}+\operatorname{Re} \boldsymbol{H}_{\text {in }} \cdot \operatorname{Re} \frac{d \boldsymbol{m}}{d t}\right\rangle\right\rangle$. The work per cycle can also be converted into an extinction cross section by dividing out the incident intensity $|E|^{2} /(2 Z)$ (where $Z$ is the impedance of the surrounding medium),

$$
\sigma_{\mathrm{ext}}=2 Z \frac{W}{|E|^{2}} \quad\left(\text { for scalar } \alpha: \quad \sigma_{\mathrm{ext}}=4 \pi k \operatorname{Im} \alpha\right) .
$$

The power per solid angle radiated by the induced dipoles in a direction specified by a unit vector $\hat{\boldsymbol{r}}$ is found by calculating the far-field Poynting vector from Eq. (1). It is composed of three terms,

$$
\frac{d P}{d \Omega}=\frac{d P_{p}}{d \Omega}+\frac{d P_{m}}{d \Omega}+\frac{k^{4}}{2 Z} \operatorname{Re}(\boldsymbol{p} \times \boldsymbol{m}) \cdot \hat{\boldsymbol{r}},
$$

The first term in Eq. (11) represents the scattered radiation of just the electric dipole $\boldsymbol{p}$, which integrates to a total scattered power given by Larmor's formula $P_{p}=\frac{4 \pi}{3 Z} k^{4}|\boldsymbol{p}|^{2}$. The second term in Eq. (11) represents the radiation pattern of just the magnetic dipole $\boldsymbol{m}$, again given by Larmor's formula. The third term, however, can completely change the radiation pattern, as it contains the interference between the fields of $\boldsymbol{p}$ and $\boldsymbol{m}$. Hence the relative phase between the induced $\boldsymbol{p}$ and $\boldsymbol{m}$ is important for the differential scattering cross section. To obtain the total scattered power, one should integrate Eq. (11) over all solid angles. The interference term integrates to 0 , as it is an odd function of $\hat{\boldsymbol{r}}$. Therefore, despite the fact that interference makes radiation patterns of magnetoelectric scatterers nontrivial, Larmor's formula for the scattered power immediately generalizes to $P=\frac{4 \pi}{3 Z} k^{4}\left(|\boldsymbol{p}|^{2}+|\boldsymbol{m}|^{2}\right)$. The optical theorem is obtained by equating scattered power $P$ to the work $W$ done by the incident field $\left(\boldsymbol{E}_{\text {in }}, \boldsymbol{H}_{\text {in }}\right)$,

$$
\begin{aligned}
& \operatorname{Im} {\left[\left(\boldsymbol{E}_{\text {in }} \boldsymbol{H}_{\text {in }}\right)^{*} \boldsymbol{\alpha}\left(\begin{array}{c}
\boldsymbol{E}_{\text {in }} \\
\boldsymbol{H}_{\text {in }}
\end{array}\right)\right] } \\
&=\frac{2}{3} k^{3}\left[\left(\boldsymbol{E}_{\text {in }} \boldsymbol{H}_{\text {in }}\right)^{*} \boldsymbol{\alpha}^{* T} \boldsymbol{\alpha}\left(\begin{array}{c}
\boldsymbol{E}_{\text {in }} \\
\boldsymbol{H}_{\text {in }}
\end{array}\right)\right],
\end{aligned}
$$

where ( $)^{*}$ indicates complex conjugate. The incident field can be eliminated by expanding $\operatorname{Im}()=\frac{1}{2 i}\left[()-()^{*}\right]$ to yield

$$
\frac{1}{2 i}\left[\boldsymbol{\alpha}-\boldsymbol{\alpha}^{* T}\right]=\frac{2}{3} k^{3} \boldsymbol{\alpha}^{* T} \boldsymbol{\alpha} .
$$

This optical theorem clearly reduces to the well-known scalar optical theorem $\operatorname{Im} \alpha=2 / 3 k^{3}|\alpha|^{2}$ in cases in which $\alpha$ is not a tensor, and can easily be converted in the Sipe-Van Kranendonk form reported by Belov, ${ }^{54}$ provided that $\boldsymbol{\alpha}$ is invertible.

\section{Addition of radiation damping to general $\alpha$ tensor}

The optical theorem (13) in itself should be viewed as a condition that can be checked for any given polarizability tensor to verify if it leads to an energy-conserving scattering theory. Given the large number of microscopic quasistatic models for metamaterial scatterers, it is unfortunate that the optical theorem in itself does not provide a recipe that specifies how to amend an electrostatic polarizability to satisfy energy conservation. In this section, we provide such a recipe. Such a method is well known for simple electric scatterers, where it is, for instance, known that an electrostatic Rayleigh polarizability $\alpha_{0}=3 V(\epsilon-1) /(\epsilon+2)$ for a small sphere of dielectric constant $\epsilon$, that in itself violates the optical theorem, ${ }^{63}$ can be amended by "addition of radiation damping." Any electrostatic $\alpha_{0}$ can be converted into a bona fide dynamic polarizability that satisfies the optical theorem by adding radiation damping ${ }^{48,49}$

$$
\frac{1}{\alpha}=\frac{1}{\alpha_{0}}-i \frac{2}{3} k^{3} .
$$

Addition of radiation damping is key in any point dipole theory for, e.g., strongly interacting plasmon particles, and has been derived on independent grounds both from the optical theorem and from size parameter expansions of dipolar scattering coefficients in Mie theory for spheroids. ${ }^{56,64}$

Here we derive a general method to add radiation damping to any electro- and magnetostatic polarizability. This allows any polarizability that is derived from a circuit model to be generalized to be a building block in an electrodynamically consistent scattering theory. We start from Eq. (12), but now assume that $\alpha$ can be diagonalized. This assumption is verified for split rings below. We call the eigenvectors $\boldsymbol{v}_{i}$, and denote the eigenvalues, which we will refer to as "eigenpolarizabilities," with $A_{i}$. Expanding the incident field at the position of the origin in the orthogonal eigenvectors

$$
\left(\begin{array}{c}
\boldsymbol{E}_{\text {in }} \\
\boldsymbol{H}_{\text {in }}
\end{array}\right)=\sum_{i} c_{i} \boldsymbol{v}_{i}
$$

and with $\boldsymbol{\alpha} \boldsymbol{v}_{i}=A_{i} \boldsymbol{v}_{i}$ and $\left\langle\boldsymbol{v}_{i} \mid \boldsymbol{v}_{j}\right\rangle=\delta_{i j}$, Eq. (12) reduces to

$$
\frac{2}{3} k^{3} \sum_{i=1}^{6}\left|c_{i}\right|^{2}\left|A_{i}\right|^{2} \geqslant \sum_{i=1}^{6}\left|c_{i}\right|^{2} \operatorname{Im} A_{i}
$$

with strict equality for lossless scatterers. Since this equation must be satisfied for any choice of incident wave (i.e., any combination of $c_{i}$ ), the generalized optical theorem for $6 \times$ 6 polarizability tensors in Eq. (13) and Ref. 54 can also be expressed in terms of the eigenpolarizabilities as

$$
\frac{2}{3} k^{3}\left|A_{i}\right|^{2} \geqslant \operatorname{Im} A_{i} \quad \forall i=1, \ldots, 6,
$$

again with strict equality for lossless scatterers. Equation (17) implies that the polarizability tensor represents an energyconserving scatterer if and only if each of its six eigenpolarizabilities are chosen to satisfy the simple scalar optical theorem $\operatorname{Im} \alpha=2 / 3 k^{3}|\alpha|^{2}$ derived for electric scatterers in Refs. 47-49 and 62 This new interpretation of the generalized optical theorem highlights the importance of two new quantities: the eigenpolarizabilities and the corresponding eigenvectors of the point scatterer polarizability. It is now evident that we 
can simply apply the scalar recipe to each eigenpolarizability separately. A compact notation for this method is

$$
\boldsymbol{\alpha}^{-1}=\boldsymbol{\alpha}_{0}^{-1}-\frac{2}{3} k^{3} i \mathbb{I} .
$$

We note that this expression, which is identical to Eq. (14) upon replacement of $1 /()$ by matrix inversion, provides a unique relation to translate a magnetostatic and electrostatic polarizability tensor $\boldsymbol{\alpha}_{0}$ derived from $L C$ circuit theory, to the corresponding electrodynamic polarizability that satisfies the optical theorem. We can hence consistently assess how intuitive ideas based on a microscopic circuit model for electrostatic and magnetostatic dipoles lead to quantitative predictions for extinction, scattering, as well as resonance hybridization, diffraction, and superradiant and subradiant damping in coupled systems, such as periodic systems, or arbitrary finite clusters.

In addition to its application to metamaterial scatterers discussed in the remainder of this paper, the formalism derived here can be used also for many other problems of current interest. For instance, Alù and Engheta have recently resolved an interesting paradox first introduced by Kerker et al. concerning the possibility of realizing zero-forward-scattering magnetodielectric nanoparticles that appear to violate the optical theorem. ${ }^{65,66}$ Alú and Engheta used a size parameter expansion of the Mie coefficients of small magnetodielectric spheres to show that magnetodielectric spheres with electric and magnetic polarizabilities that are almost equal in magnitude but opposite in sign have a highly anisotropic scattering pattern, and violate the optical theorem unless one includes size expansion terms beyond the quasistatic limit. We note that all the essential physics of this problem can also be studied with ease using our formalism. It is easy to verify that a diagonal isotropic tensor with $\alpha_{E}=-\alpha_{H}$ indeed corresponds to a spherical scatterer with a highly anisotropic radiation pattern according to Eq. (11), with very small forward scattering. If radiation damping is added as in Eq. (18), the optical theorem is indeed satisfied, and forward scattering is low but not zero, in accordance with Ref. 65. First, this simple example shows that magnetoelectric point dipoles can have counterintuitive properties, such as highly anisotropic radiation patterns, very much unlike simple electric dipoles. Secondly, our formalism provides an easy analytical model, which does not need a size parameter expansion of an exact solution, to study or design how far the zero-forward scattering paradox extends to anisotropic particles that also have off-diagonal, magnetoelectric elements.

\section{POLARIZABILITY OF SPLIT RING RESONATORS}

\section{A. Symmetry}

As an example of our general theory, we consider the specific example of split ring resonators. The quasistatic polarizability of split ring resonators was discussed in detail by Garcìa-Garcìa et al. ${ }^{58}$ and also analyzed by Belov et al., ${ }^{50}$ Shamonina et al., ${ }^{36}$ Gorkunov et al., ${ }^{38}$ and Marqués et al. ${ }^{39}$ We consider the $L C$ resonance of an infinitely thin split ring in the $x y$ plane, with split oriented along the $x$ axis, as shown in Fig. 1(a). Such a split ring can only have an electric dipole moment along $x$ and a magnetic dipole moment along $z$. As outlined in Ref. 58, the only nonzero tensor elements are $\alpha_{E E}^{x x}$, $\alpha_{H H}^{z z}$, and the cross-coupling terms $\alpha_{H E}^{z x}, \alpha_{E H}^{x z}$,

$$
\boldsymbol{\alpha}_{\mathrm{SRR}}=\left(\begin{array}{ccccc}
\alpha_{E E}^{x x} & 0 & \cdots & 0 & \alpha_{E H}^{x z} \\
0 & & & & 0 \\
\vdots & & \ddots & & \vdots \\
0 & & & & 0 \\
\alpha_{H E}^{z x} & 0 & \cdots & 0 & \alpha_{H H}^{z z}
\end{array}\right)
$$

The symmetry constraints that set which elements of $\boldsymbol{\alpha}_{\mathrm{SRR}}$ are zero are valid both for the electrodynamic and quasistatic polarizability of split rings.

\section{B. Quasistatic $R L C$ model}

We now construct the electrodynamic polarizability by starting from a quasielectrostatic and magnetostatic polarizability derived from a single resonant $R L C$ equation of motion. Therefore, we take a common resonant frequency dependence out of the tensor elements, writing

$$
\boldsymbol{\alpha}_{\mathrm{SRR}}^{\text {static }}=\alpha(\omega)\left(\begin{array}{ccccc}
\eta_{E} & 0 & \cdots & 0 & i \eta_{C} \\
0 & & & & 0 \\
\vdots & & \ddots & & \vdots \\
0 & & & & 0 \\
-i \eta_{C} & 0 & \cdots & 0 & \eta_{H}
\end{array}\right),
$$

where $\eta_{E}, \eta_{C}$, and $\eta_{H}$ are constant and $\alpha(\omega)$ is a Lorentzian prefactor,

$$
\alpha(\omega)=\frac{\omega_{0}^{2} V}{\omega_{0}^{2}-\omega^{2}-i \omega \gamma} .
$$

Here, $\omega_{0}$ is the SRR resonance frequency $\omega_{0} \approx \frac{1}{\sqrt{L C}}, \gamma$ is the damping rate due to the Ohmic loss of gold, and $V$ is the physical particle volume. As in the plasmonic case, this approximation is coined "quasi"-static, as it does contain frequency $\omega$, but it does not contain the velocity of light $c$. In this formulation, all the frequency dependence and the units of $\boldsymbol{\alpha}_{\mathrm{SRR}}$ are contained in $\alpha(\omega)$. The parameters $\eta_{E}, \eta_{H}$, and $\eta_{C}$ are dimensionless. For a lossless split ring, $\eta_{E}, \eta_{H}$, and $\eta_{C}$ are all real and assumed positive on the basis of the anticipated in-phase response at very low frequencies. ${ }^{58}$ The $i$ multiplying $\eta_{C}$ is needed to ensure lossless scattering (for $\gamma=0$ ), and can be understood microscopically as reflecting the fact that any charge separation that is induced through a current lags the current by a quarter wave (and vice versa). In our model, all Ohmic losses are introduced via $\gamma$.

\section{Limit on magnetoelectric coupling}

Having constructed a quasistatic polarizability in accordance with $R L C$ circuit models proposed in earlier reports, we apply radiation damping according to Eq. (18) to obtain a scatterer that has a correct energy balance: ${ }^{67}$

$$
\boldsymbol{\alpha}_{\mathrm{SRR}}^{-1}=\left(\boldsymbol{\alpha}_{\mathrm{SRR}}^{\text {static }}\right)^{-1}-\frac{2}{3} k^{3} \mathbb{I} .
$$

So far we have not explicitly discussed absorption loss, except through the inclusion of the material damping constant $\gamma$ in the quasistatic polarizability. Starting from a quasistatic 
polarizability with quasistatic eigenpolarizabilities $A_{i}^{\text {static }}$, the albedo for each eigenillumination $\boldsymbol{v}_{i}$ can be expressed as

$$
a_{i}=\frac{1}{1+\frac{2}{3} k^{3} \operatorname{Im} A_{i}^{\text {static }}} .
$$

This albedo quantifies the fraction of energy absorbed in the scatterer compared to the total energy extinct by the scatterer when illuminated at eigenillumination. It follows that for any lossy scatterer, the imaginary part of each eigenvalue $A_{i}^{\text {static }}$ of the quasistatic polarizability tensor must be positive to ensure $0 \leqslant a \leqslant 1$. In the case of a tensorial $\boldsymbol{\alpha}$ with loss included as in Eqs. (20) and (21), one needs to explicitly verify that each eigenvalue has a positive imaginary part. The eigenvalues of Eq. (20) are $A_{ \pm}^{\text {static }}=\alpha(\omega) \lambda_{ \pm}$with $\lambda_{ \pm}=$ $1 / 2\left[\eta_{E}+\eta_{H} \pm \sqrt{\left(\eta_{E}-\eta_{H}\right)^{2}+4 \eta_{C}^{2}}\right]$. Since $\operatorname{Im} \alpha(\omega) \geqslant 0$ and $\lambda_{ \pm}$are real, we find that both eigenvalues have a positive imaginary part only if both $\lambda_{+}$and $\lambda_{-}$are positive. Thus, loss sets an additional constraint on the polarizability tensor, and limits the magnetoelectric coupling to

$$
\left|\eta_{C}\right| \leqslant \sqrt{\eta_{E} \eta_{H}}
$$

This result implies a very important limitation on magnetoelectric scatterers: it states that a magnetoelectric cross coupling $\left(\eta_{C}\right)$ can only be generated if there is a sufficiently strong directly electric and directly magnetic response. We note that this constraint is very similar to the constraint on the magnetoelectric cross coupling in constitutive tensors derived for homogeneous bi-anisotropic media in Ref. 53 that recently attracted attention in the framework of proposals for repulsive Casimir forces. ${ }^{68,69}$ While our derivation was specific for split rings, we note that similar constraints hold for all magnetoelectric scatterers. In the presence of material loss, the magnetoelectric coupling terms are limited by the fact that all quasistatic eigenpolarizabilities must have a positive imaginary part.

\section{PREDICTED SCATTERING PROPERTIES OF SINGLE SPLIT RINGS}

In the remainder of the paper, we discuss some insights that the proposed magnetoelectric point scattering theory provides in how split rings scatter. In this section, we will consider the eigenmodes and the radiation patterns of a single SRR for $\boldsymbol{\alpha}$ given by Eq. (22). Next, we predict which set of experiments will provide full information on the elements of the polarizability tensor. We will show how the extinction cross sections can be translated back to retrieve SRR polarizabilities and magnetoelectric cross polarizabilities of a single SRR. Although the results we present are general, we use a specific set of parameters for all the figures presented in this paper. These parameters are chosen to fit to the properties of split rings that are resonant at $\lambda=1.5 \mu \mathrm{m}\left(\omega_{0} / 2 \pi=200 \mathrm{THz}\right)$ and that consist of $200 \times 200 \mathrm{~nm}$ gold split rings with a thickness of $30 \mathrm{~nm}$ and a gap width of $90 \mathrm{~nm}$. Thus we take $V=200 \times 200 \times 30 \mathrm{~nm}^{3}$. We set the damping rate to be that of gold $\gamma=1.25 \times 10^{14} \mathrm{~s}^{-1}$ as fitted to optical constants tabulated in Ref. 70. We use $\eta_{E}=0.7, \eta_{H}=0.3$, and $\eta_{C}=0.4$. These parameters were chosen because (a) they reproduce quantitatively the extinction cross section under normal incidence along the $z$ axis measured by Husnik et al., ${ }^{17}$ and (b) they fit well to our transmission data on arrays of different densities of split rings taken at normal incidence $^{9}$ and as a function of incidence angle. ${ }^{71}$ The chosen values correspond to on-resonance polarizabilities $\alpha_{E E}=$ $4.6 \mathrm{~V}, \alpha_{H H}=2.1 \mathrm{~V}$, and $\alpha_{E H}=2.5 \mathrm{~V}$, all well in excess of the physical SRR volume $V$ as is typical for strong scatterers. Finally, we note that the calculated albedo fits well to the albedo $a=0.5-0.75$ calculated by FDTD by Husnik et al. ${ }^{17}$

\section{A. Radiation patterns and eigenvectors of the polarizability tensor}

In Fig. 1, we consider the eigenstates of the split ring polarizability tensor presented in Eq. (22). We first assume

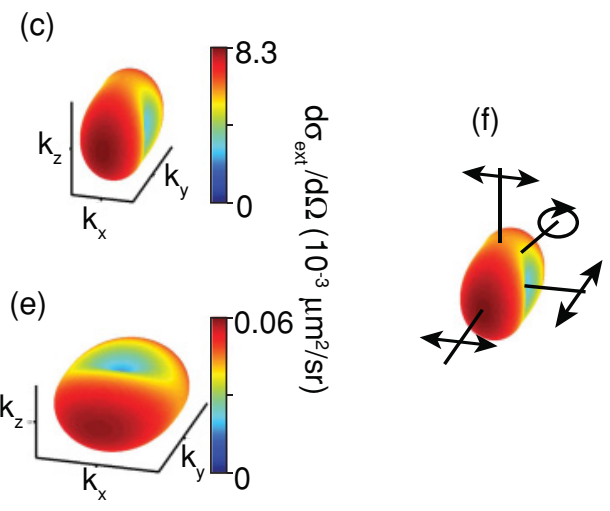

FIG. 1. (Color online) Split ring radiation patterns corresponding to the polarizability tensor eigenvectors. (a) (Sketch) A single split ring resonator can have an electric dipole moment $\boldsymbol{p}$ along the $x$ axis due to charging of the split. Circulating current $j$ in the ring gives rise to a magnetic dipole moment $\boldsymbol{m}$ in the $z$ direction. (b),(c) Radiation patterns of the two eigenmodes of an SRR in the case of no off-diagonal magnetoelectric coupling $\left(\eta_{E}=0.7, \eta_{H}=0.3, \eta_{C}=0\right)$. The electric dipole moment oriented along the $x$ axis radiates most of its amplitude in the $k y, k z$ plane, while the magnetic dipole oriented along the $z$ axis radiates mostly into the $k x, k y$ plane. (d),(e) Radiation patterns of the eigenvectors with magnetoelectric cross coupling $\left(\eta_{C}=0.4\right)$. (f) Indication of the polarization of the light radiated by the eigenvector with a largest eigenvalue [(c)]. Light is linearly polarized for wave vectors along the Cartesian axes, but elliptically polarized in general. The direction of strongest circular dichroism in extinction and scattering is in the $x z$ plane. 
that the cross-coupling terms are absent, i.e., $\eta_{C}=0$, in which case the polarizability tensor is diagonal, with eigenpolarizabilities $\alpha(\omega) \eta_{E}$ and $\alpha(\omega) \eta_{H}$. The corresponding orthogonal eigenmodes are $\left(p_{x}, m_{z}\right)=(1,0)$ and $\left(p_{x}, m_{z}\right)=(0,1)$. Figures 1 (b) and 1(c) show radiation patterns of the two eigenmodes. Figure 1(b) shows the radiation pattern of the purely electric eigenmode $\left(p_{x}, m_{z}\right)=(1,0)$, and Fig. $1(\mathrm{c})$ shows the radiation pattern of the purely magnetic eigenmode $\left(p_{x}, m_{z}\right)=(0,1)$. Note that both $\boldsymbol{p}_{x}$ and $\boldsymbol{m}_{z}$ radiate as simple dipoles with a $\sin ^{2} \theta$ far-field radiation pattern. ${ }^{72}$ The two eigenmodes can be selectively excited by impinging with a plane wave incident along the $z$ axis with an $x$-polarized $E$ field (electric eigenmode), or with a plane wave incident along the $x$ axis with $y$ polarization ( $z$-polarized $H$ field, magnetic eigenmode). The extinction cross section of a single split ring at these two incidence conditions is set by $\sigma_{\text {ext }}=4 \pi k \operatorname{Im}\left(\alpha_{E E}\right)$ and $\sigma_{\text {ext }}=4 \pi k \operatorname{Im}\left(\alpha_{H H}\right)$.
Next, we consider extinction and eigenmodes for arbitrary values of the cross coupling. It is easy to see that the extinction cross section at the two special illumination conditions (incident along $z$ - and $x$-polarized and incident along $x$ with $y$ polarization, respectively) remain equal to $\sigma_{\text {ext }}=4 \pi k \operatorname{Im}\left(\alpha_{E E}\right)$ and $\sigma_{\text {ext }}=4 \pi k \operatorname{Im}\left(\alpha_{H H}\right)$. However, for nonzero $\eta_{C}$, these incidence conditions and polarizabilities no longer correspond to the eigenvalues and eigenvectors of the polarizability tensor, which now have mixed magnetoelectric character. In the extreme case of strongest magnetoelectric coupling $\left(\eta_{C}=\sqrt{\eta_{E} \eta_{H}}\right)$, the eigenvectors reduce to $\left(p_{x}, m_{z}\right)=\left(1, i \sqrt{\eta_{E} / \eta_{H}}\right)$ and $\left(p_{x}, m_{z}\right)=\left(1,-i \sqrt{\eta_{H} / \eta_{E}}\right)$. The associated far-field radiation patterns of these eigenvectors correspond to coherent superpositions of the radiation pattern of an $x$-oriented electric dipole and a $z$-oriented magnetic dipole, with a quarter wave phase difference. Figures 1(d) and 1(e) show the on-resonance radiation pattern, assuming

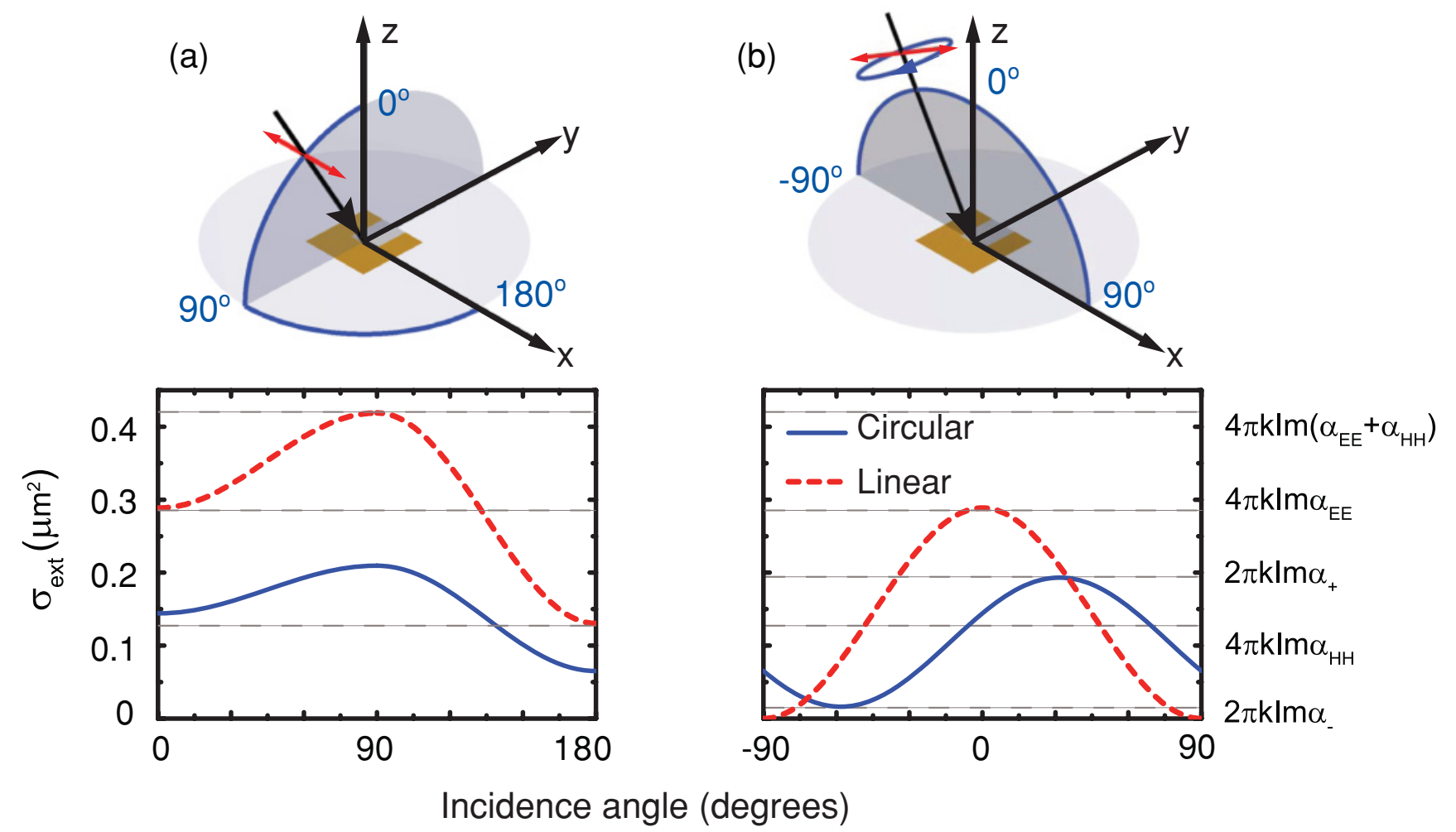

FIG. 2. (Color online) We calculate the extinction cross section $\sigma_{\text {ext }}$ as a function of illumination angle and polarization. In panel (a), we sweep the incident wave vector over a total angular range of $180^{\circ}$, over a trajectory [see panel (a), top] starting from the $z$ axis (labeled $0^{\circ}$ ) to the $-y$ axis (labeled $90^{\circ}$ ), and then through the $x y$ plane to the $x$ axis (full $180^{\circ}$ ). The red dashed line represents $\sigma_{\text {ext }}$ for linearly polarized incident illumination, where in (a) the polarization is kept in the $x y$ plane as shown (i.e., polarization is along $x$ for the first $90^{\circ}$ of the trajectory, rotating continuously to polarization vector along $y$ in the second part of the trajectory). The blue solid curve represents extinction for right-handed circularly polarized illumination, which for the incidence conditions in (a) is half the extinction seen for linearly polarized light. For the trajectory of incident wave vectors in panel (a), $\sigma_{\text {ext }}$ at normal incidence to the split ring is a measure for only $\alpha_{E E}$ as $E_{x}$ is the only driving field. Increasing the angle to $90^{\circ}$, both polarizations $E_{x}$ and $H_{z}$ excite the dipoles in the SRR, so $\sigma_{\text {ext }}$ is a measure for the sum of the terms on the diagonal of the polarizability tensor $\left(\alpha_{E E}+\alpha_{H H}\right)$. Changing the incidence condition to $180^{\circ}$ removes the $E_{x}$ component of the field, leaving only $H_{z}$ of the incident light to drive the SRR. Hence $\sigma_{\text {ext }}$ at $180^{\circ}$ in panel (a) is a measure for purely $\alpha_{H H}$. In panel (b) we sweep the incident wave vector from the $-x$ axis (labeled $-90^{\circ}$ ) via the $z$ axis (again labeled $0^{\circ}$ ) to the $+x$ axis (labeled $90^{\circ}$ ). The color- and linetype coding is as in panel (a). However, now we constrain the linear polarization to have no $y$ component. Panel (b) $\sigma_{\text {ext }}$ shows that as a function of the incident angle in the $x z$ plane for right-handed circular polarization, minima and maxima in $\sigma_{\text {ext }}$ occur as a function of angle, which are a measure for the eigenpolarizabilities $\alpha_{-}$and $\alpha_{+}$, respectively. Both sets of measurements in panels (a) and (b) together provide information on all the components of the polarizability tensor, $\alpha_{E E}, \alpha_{H H}$, and $\alpha_{E H}$. 
$\eta_{E}=0.7, \eta_{H}=0.3$, and $\eta_{C}=0.4$. Note that these parameters are close to the limit of strongest possible magnetoelectric coupling. Figures $1(\mathrm{~d})$ and $1(\mathrm{e})$ reveal that the radiation pattern of each eigenmode is nondipolar. Rather than a $\sin ^{2} \theta$ donut-shaped pattern, an elongated radiation pattern occurs, with maximum extent in the $y$ direction. The polarization in the far field is linear for directions along the Cartesian axis, but is generally elliptical.

\section{B. Extinction cross sections to measure polarizability}

Figure 2 shows the extinction cross section predicted by our point scattering model of a single split ring for different incidence conditions. In Fig. 2(a), the incident wave vector is swept from the $z$ direction to the $y$ direction while maintaining $x$-polarized light. For this set of incidence conditions, the resulting extinction cross sections only depend on $\alpha_{E E}$ and $\alpha_{H H}$, and are entirely independent of the off-diagonal coupling strength $\alpha_{E H}$. The cross section increases from $\sigma_{\mathrm{ext}}=$ $4 \pi k \operatorname{Im} \alpha_{E E}$ as the split ring is only driven by the incident $E_{x}$ field when light is incident along $z$, to $\sigma_{\text {ext }}=4 \pi k\left(\operatorname{Im} \alpha_{E E}+\right.$ $\left.\operatorname{Im} \alpha_{H H}\right)$ as the split ring is driven by the incident $E_{x}$ field plus the incident $H_{z}$ field. When the wave vector is rotated to the $x$ axis, the extinction cross section diminishes to $4 \pi k \operatorname{Im} \alpha_{H H}$, as the split ring is only driven by $H_{z}$. The chosen values $\eta_{E}=$ $0.7, \eta_{H}=0.3$, and $\eta_{C}=0.4$ that we also used for Figs. 1(d) and 1 (e) yield extinction cross sections $\sigma_{\text {ext }}=4 \pi k \operatorname{Im} \alpha_{E E}=$ $0.29 \mu \mathrm{m}^{2}$ and $\sigma_{\text {ext }}=4 \pi k \operatorname{Im} \alpha_{H H}=0.13 \mu \mathrm{m}^{2}$. The predicted $\sigma_{\text {ext }}=4 \pi k \operatorname{Im} \alpha_{E E}=0.29 \mu \mathrm{m}^{2}$ is consistent with the measurement $\left(\sigma_{\text {ext }}=0.3 \mu \mathrm{m}^{2}\right)$ reported by Husnik et al. ${ }^{17}$ It is important to note that measurements along Cartesian incidence directions and with linear Cartesian polarizations yield only the diagonal elements of the polarizability tensor. Indeed, the proposed measurements form a redundant set of measurements of $\alpha_{E E}, \alpha_{H H}$, and $\left(\alpha_{E E}+\alpha_{H H}\right)$, but do not provide any insight into the magnetoelectric cross coupling in the electrodynamic polarizability tensor. ${ }^{73}$

To measure the eigenpolarizabilities, it is necessary to selectively address the eigenvectors of the polarizability tensor. As noted above, the eigenvectors in the case of strong magnetoelectric coupling $\eta_{C} \approx \sqrt{\eta_{E} \eta_{H}}$ tend to $\left(p_{x}, m_{z}\right)=$ $\left(1, i \sqrt{\eta_{E} / \eta_{H}}\right)$ and $\left(1,-i \sqrt{\eta_{H} / \eta_{E}}\right)$. These eigenvectors require simultaneous driving by $E_{x}$ and $H_{z}$, with a quarter wave phase difference. We note that such fields can be generated by circularly polarized light with incident wave vector constrained to the $x z$ plane. Indeed, at maximally strong magnetoelectric coupling and $\eta_{E}=\eta_{H}$, circularly polarized light incident at $45^{\circ}$ from the $z$ axis would selectively excite exactly one eigenmode. Therefore, we expect angle-resolved extinction measurements for oppositely handed circularly polarized beams to reveal the eigenpolarizabilities. Figure 2(b) plots the extinction cross section for right-handed circular polarization, as a function of the angle of incidence in the $z$ plane, for illumination tuned to the $L C$ resonance frequency. Naturally, at normal incidence the extinction is exactly half the extinction obtained for linear polarization, as a consequence of the fact that $E_{y}$ does not interact with the split ring at all. Strikingly, the extinction cross section is predicted to behave asymmetrically as a function of incidence angle. The extinction increases when going to a positive angle and decreases when going to a negative angle. Changing handedness is equivalent to swapping positive and negative angles. A detailed analysis shows that the maximum in extinction corresponds to the largest eigenvalue of the polarizability tensor $\left(\sigma_{\mathrm{ext}}=2 \pi k \operatorname{Im} \alpha_{+}\right)$, while the minimum in extinction corresponds to the smallest eigenvalue $\left(\sigma_{\text {ext }}=2 \pi k \operatorname{Im} \alpha_{-}\right)$. Therefore, circularly polarized measurements reveal the eigenvalues of the polarizability tensor. Combining such circularly polarized extinction measurements with the measurements under Cartesian incidence in Fig. 2(a), therefore, allows us to extract all components of the polarizability tensor. In addition to the contrast in extinction, the angle at which the maximum circular dichroism occurs is a second, independent measure for the magnetoelectric coupling strength. The measurements in Figs. 2(a) and 2(a) together hence provide full, even redundant, information on $\eta_{E}, \eta_{H}$, and $\eta_{C}$.

\section{Structural chirality}

The results plotted in Fig. 2(b) show that magnetoelectric coupling in the $6 \times 6$ polarizability tensor directly implies structural chirality. It is exhilarating that this interesting phenomenon first reported by Refs. 27 and 31 for the transmission of arrays of scatterers is naturally present in the theory. However, while previous analysis of structural chirality focused on transmission through periodic arrays, we predict that circular dichroism already appears in the extinction cross section of a single split ring, with a strength set by how close the magnetoelectric coupling strength is to its limit $\sqrt{\eta_{E}, \eta_{H}}$. Here we use the term "circular dichroism in extinction" not to refer to differential absorption of left and right circular polarization as in usual circular dichroism, but to differential extinction. Indeed, the circular dichroism in extinction is a difference in extinction cross section for left and right circularly polarized light that occurs independently of whether there is material loss, as opposed to, e.g., asymmetric transmission phenomena through arrays, which are claimed to require dissipation. ${ }^{31}$ For maximally magnetoelectrically coupled systems, the smallest eigenvalue is identically zero, implying that such a scatterer is transparent for one circular polarization, and achieves its strongest scattering for the opposite handedness. We expect that our $6 \times 6$ polarizability tensor can be successfully used to describe all structurally chiral scatterers reported today, as well as clusters and periodic arrays thereof.

\section{A COUPLED SYSTEM: SPLIT RING DIMERS}

So far, this paper has focused purely on the scattering properties of single magnetoelectric point scatterers. In the remainder of the paper, we illustrate that our method can be easily used to analyze multiple scattering by magnetoelectric scattering clusters. To calculate the response of a system of coupled magnetoelectric dipoles, we generalize the general self-consistent equation that describes scattering of clusters of electric dipoles $\boldsymbol{p}$ as reviewed in Ref. 49. Assuming a system of $N$ magnetoelectric point scatterers situated at positions $\boldsymbol{r}_{1}, \ldots, \boldsymbol{r}_{N}$, the response upon illumination by an incident field $\left(\boldsymbol{E}_{\text {in }}(\boldsymbol{r}), \boldsymbol{H}_{\text {in }}(\boldsymbol{r})\right)$ is determined by a set of $N$ self-consistent equations for the induced dipole moments in each scatterer. The dipole moment induced in scatterer $n$ with polarizability 
tensor $\boldsymbol{\alpha}_{n}$ is

$$
\begin{aligned}
&\left(\begin{array}{c}
\boldsymbol{p}_{n} \\
\boldsymbol{m}_{n}
\end{array}\right)=\boldsymbol{\alpha}_{n}\left[\left(\begin{array}{c}
\boldsymbol{E}_{\mathrm{in}}\left(\boldsymbol{r}_{n}\right) \\
\boldsymbol{H}_{\mathrm{in}}\left(\boldsymbol{r}_{n}\right)
\end{array}\right)\right. \\
&\left.+\sum_{\substack{q=1, \ldots, N \\
q \neq n}} \boldsymbol{G}^{0}\left(\boldsymbol{r}_{n}, \boldsymbol{r}_{q}\right)\left(\begin{array}{c}
\boldsymbol{p}_{q} \\
\boldsymbol{m}_{q}
\end{array}\right)\right] .
\end{aligned}
$$

Using this equation, we can attempt to reinterpret recent measurements that evidence significant coupling in split rings in two-dimensional (2D) arrays, as well as in oligomers. ${ }^{9,24,34,42}$ Here we focus on the extinction of a dimer of split rings in a so-called "stereodimer" configuration, first studied by Liu et al. ${ }^{34}$ Figure 3 shows such a "stereodimer," consisting of two SRR's in vacuum $\left(V=200 \times 200 \times 30 \mathrm{~nm}^{3}\right.$, resonant at a wavelength around $1500 \mathrm{~nm}$ ), both parallel to the $x y$ plane, vertically stacked with a small height difference of $150 \mathrm{~nm}$. The upper SRR is rotated by a twist angle $\psi$ around the $z$ axis. On the basis of the report by Liu et al., ${ }^{34}$ we expect two resonance peaks with an angle-dependent splitting, which can be explained in an $L C$ model as the summed effect of electric dipole-dipole coupling and magnetic dipole-dipole coupling.

We calculate the extinction versus twist angle and wavelength of an incident beam incident from the $+z$ direction, with $x$ polarization. This beam directly excites $\boldsymbol{p}_{x}$ in both rings, which also drive each other. We first analyze the experiment assuming that there is no magnetoelectric coupling term (setting $\eta_{C}=0$, although we keep $\eta_{E}=0.7$ and $\eta_{H}=0.3$ ). As Fig. 3(b) shows, the extinction shows a single strong resonance that is blueshifted relative to the single SRR resonance at $200 \mathrm{THz}$. As a function of twist angle, this broad resonance redshifts to $200 \mathrm{THz}$ at a twist of $90^{\circ}$, and shifts back to $220 \mathrm{THz}$ at a twist of $180^{\circ}$. There is no sign of a second resonance, which might be hidden below the strong resonance. To bring out the second resonance more clearly, we reduce the loss in Fig. 3(b) to a 10-times-lower value $\gamma=1.25 \times 10^{13} \mathrm{~s}^{-1}$ for gold in Figs. 3(c) and 3(d). For this almost absorption-free system, Fig. 3(c) indeed shows two resonances in extinction. The blueshifted resonance is now observed to cross with a narrow redshifted resonance. The crossing is symmetric around $90^{\circ}$ and is consistent with the hybridization of an electric dipole fixed along $x$, with a second one above it twisted by an amount $\psi$. The two branches have a very different width and strength, consistent with the fact that a symmetric configuration of dipoles couples more strongly to external fields (blueshifted resonance) than an antisymmetric "dark" configuration (redshifted resonance).

To verify whether the two resonances observed in Fig. 3(a) are all resonances in the system, we change the angle of incidence to $45^{\circ}$ in the $x z$ plane, so that the exciting field has an $H_{z}$ component to drive the split rings, in addition to an $E_{x}$ component. Figure 3(d) shows that in this case, four resonances occur in extinction. In addition to the two curved bands excited by $E_{x}$, there are also two nondispersive bands with a twist-independent splitting. Obviously, these bands are due to the coupling of two magnetic dipoles in symmetric (broad and intense band) and antisymmetric head-to-tail configuration. The existence of four instead of two modes is a new insight compared to $L C$ circuit models, ${ }^{34,44}$ but is logical in view of the fact that split rings have both a magnetic and an electric response, which are decoupled under the assumption $\eta_{C}=0$.

Next we analyze the extinction in the presence of magnetoelectric coupling, setting $\eta_{C}=0.4$. Again, we first examine the extinction in the presence of realistic loss $(\gamma=1.25 \times$ $10^{14} \mathrm{~s}^{-1}$ ) for gold in Fig. 3(e). As also predicted by FDTD simulations by Liu et al., ${ }^{34}$ there appear to be two bands. The blueshifted band is again very broad, but now has a frequency shift away from the single SRR resonance that is significantly larger for twist angle $180^{\circ}$ than for $0^{\circ}$. These effects were explained by Liu et al. ${ }^{34}$ as due to an additive (subtractive) correction to the dominant electric hybridization at twist angle $180^{\circ}\left(0^{\circ}\right)$ that occurs due to magnetic dipole coupling. A surprise is that the diagram is not symmetric anymore around $90^{\circ}$ twist as in the case of zero magnetic coupling. Instead, the extinction appears to show an anticrossing at a twist angle $60^{\circ}$ These features were also predicted by FDTD simulations by Liu et al. ${ }^{34}$ However, the presence of an anticrossing at twist angle $\psi=60^{\circ}$ could not be interpreted by Liu et $a l{ }^{34}$ within an $L C$ static circuit model, except by invoking higher-order multipolar corrections. Here we see that a purely dipolar model may also explain all features of the experiment provided that magnetoelectric coupling is accounted for. While we do not claim that multipolar effects are not present in actual experiments, it is an important insight that split ring polarizabilities with magnetoelectric coupling terms may provide much richer physics then expected from quasistatic circuit theory. A main advantage of point dipole theory is that the underlying mode structure does not need to be recouped from FDTD simulations, but is easily resolved by repeating a calculation of extinction cross sections with low loss (as done in Fig. 3), or by analyzing the poles of the coupling matrix in Eq. (25) that relates $(\boldsymbol{p}, \boldsymbol{m})$ to $\left(\boldsymbol{E}_{\mathrm{in}}, \boldsymbol{H}_{\mathrm{in}}\right)$. The computational effort for $N$ split rings is equivalent to diagonalizing or inverting a $6 N \times 6 N$ matrix.

To more clearly bring out all the resonances, we artificially reduce the damping $\gamma=1.25 \times 10^{13} \mathrm{~s}^{-1}$ to ten times less than the damping of gold, and plot the response of the system under normal incidence (f) and $45^{\circ}$ incidence (g) in Figs. 3(f) and $3(\mathrm{~g})$. The anticrossing at twist angle $\psi=60^{\circ}$ appears to be due to the coupling of four modes, as opposed to the intuition from $L C$ circuit theory that only two resonances anticross. The existence of four rather than two modes in a split ring dimer appears surprising and is a second indication of the rich physics of magnetoelectric scatterers. Intuition from $L C$ circuits is that although the subspace of driving fields is two-dimensional ( $E_{x}$ and $H_{z}$ ), nonetheless only one mode per split ring exists. The usual reasoning in $L C$ models is that the relation between electric and magnetic dipole moment is completely fixed and independent of driving, since the loop current and accumulated charge are directly related. Such a constraint is not general: in electrodynamic multipole expansions, magnetic polarizabilities are determined independently from the electric ones. The intuition from $L C$ theory that there is only one mode per scatterer is only retrieved in our model right at the limit of strongest magnetoelectric coupling $\eta_{C}=\sqrt{\eta_{E} \eta_{H}}$, since in that case one polarizability is identically zero. We note that the values $\eta_{E}=0.7, \eta_{H}=0.3$, and $\eta_{C}=0.4$ used in this work (that we fitted to our angle-resolved transmission experiments on $200 \times 200 \mathrm{~nm} \mathrm{Au} \mathrm{split} \mathrm{rings} \mathrm{on} \mathrm{glass)} \mathrm{are} \mathrm{close} \mathrm{to} \mathrm{the}$ 
(a)
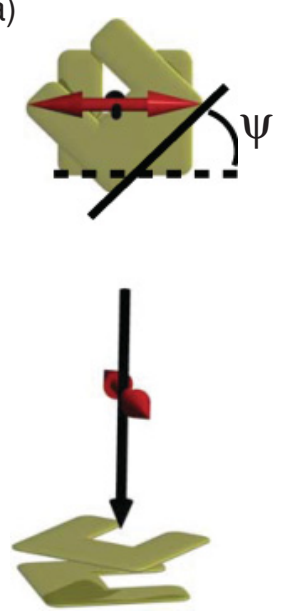
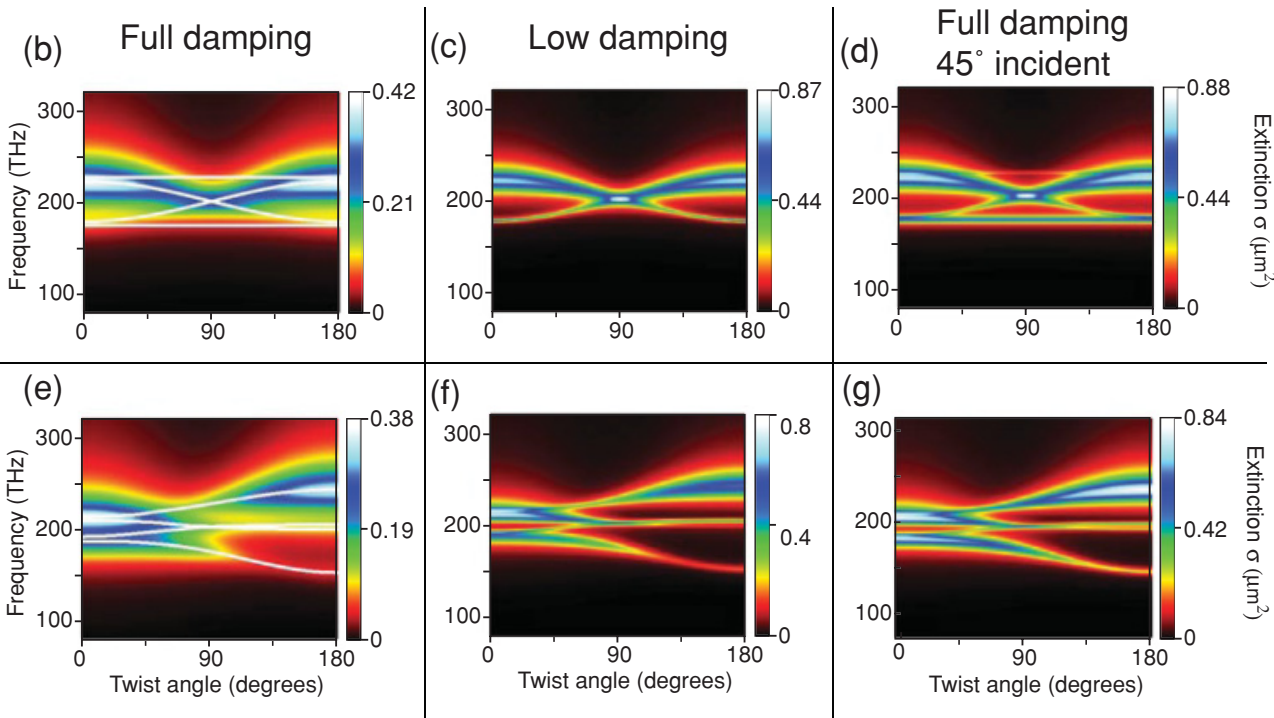

FIG. 3. (Color online) Extinction cross sections $\sigma_{\text {ext }}$ vs frequency and twist angle for an SRR stereodimer structure. Panel (a) shows the geometry (top view and side view) in which two SRR's are vertically stacked. The upper SRR is rotated around the $z$ axis by the twist angle $\psi$. We calculate extinction for light impinging from the $z$ direction with polarization along $x$, i.e., along the base of the lower SRR in (b), (c), (e), and (f). In (d) and (g), we use $45^{\circ}$ incidence in the $x z$ plane, so that the $\boldsymbol{H}$ field of the excitation light directly couples also to the magnetic polarizability. Panels (b), (c), and (d) show extinction assuming no cross-coupling term $\left(\eta_{C}=0\right)$, while (e), (f), and (g) show extinction assuming strong magnetoelectric coupling $\left(\eta_{C}=0.4\right)$. Panels (b) and (e) assume the damping rate of gold $\gamma=1.25 \times 10^{14} \mathrm{~s}^{-1}$. To more clearly bring out the four mode structure, we reduce the damping tenfold for the calculations in (c), (d), (f), and (g). There are four modes present in the system. White lines in (b) and (e) indicate the frequencies of the modes, as taken from the resonances in the low-damping case, i.e., the resonances in panels $(\mathrm{d})$ and $(\mathrm{g})$.

limit of strong magnetoelectric coupling. Whether a general argument exists why physical scatterers are or are not exactly at the limit of strongest magnetoelectric coupling $\eta_{C}=\sqrt{\eta_{E} \eta_{H}}$ is a question beyond the scope of this paper.

\section{CONCLUSION}

In conclusion, we have developed a multiple scattering theory by means of which we can calculate scattering and extinction for any magnetoelectric scatterer with known polarizability tensor, as well as for arbitrary finite clusters. As opposed to $L C$ circuit models, our model obeys energy conservation, contains all interference effects, and allows quantitative prediction of absolute cross sections, spectral linewidths, and line shapes. While beyond the scope of this paper, the theory is readily extended to deal with arbitrary periodic lattices by generalizing Ewald lattice sums ${ }^{49}$ to deal with both $\boldsymbol{E}$ and $\boldsymbol{H}$. Since the electrodynamic polarizability tensor can be directly constructed from quasistatic circuit theory, we expect that our model is readily applicable to many current experiments using chiral and nonchiral metamaterial building blocks for which quasistatic models have been proposed.

Our model does not give any insight into whether the response of a given structure is truly dipolar. Also, our model does not provide any insight or quantitative predictions based on microscopic considerations for the magnitude of the polarizability. For such microscopic considerations, based on, e.g., current density distributions derived from full wave simulations, we refer the reader to Refs. 18-20,43,45,46. Rather, our model allows one to verify if specific data or microscopic calculations are consistent at all with point dipole interactions, allowing to verify or falsify common intuitive explanations in the literature that have so far been based on quasistatic considerations. Also, our model allows one to assess if a single polarizability tensor indeed can describe a range of different experiments with, e.g., split ring clusters, as should be expected from a consistent model. Finally, our model is the simplest electrodynamic model to consistently describe how metamaterials and photonic crystals are formed from magnetoelectric scatterers. A first step is to confirm the parameters used in this work for $\eta_{E}, \eta_{H}$ and $\eta_{C}$ by targeted experiments. While the value for $\eta_{E}$ used in this work is consistent with the extinction cross section measured by Husnik et al., ${ }^{17}$ we propose that the new insight that magnetoelectric coupling is far stronger than the magnetic polarizability be confirmed by off-normal circularly polarized extinction measurements as proposed in Sec. IV.

The most important property of our theory is that a polarizability tensor validated for a single scatterer can readily be used to predict all quantitative scattering properties of composite lattices and antennas. We hence expect that new insights can be obtained in effective-medium constants of metamaterial arrays. Our analytical model not only facilitates design, but will also allow us to determine rigorously whether, even in the ideal case (no loss, no multipole corrections), metamaterial building blocks can give rise to a desired $\epsilon$ and $\mu$, despite the large importance of electrodynamic corrections. ${ }^{7,9,74}$ In addition to generating new insights for metamaterials, our theory also opens new design routes for gratings and antennas with unprecedented polarization properties. As an example, in this paper we analyzed the four-mode anticrossing due 
TABLE I. Conversion between SI units and the unit system used throughout this paper.

\begin{tabular}{lcc}
\hline \hline & & \\
Quantity & Symbol & relation to SI \\
\hline Electric field & $\boldsymbol{E}$ & $\boldsymbol{E}_{\mathrm{SI}}$ \\
Magnetic field & $\boldsymbol{H}$ & $Z \boldsymbol{H}_{\mathrm{SI}}$ \\
Electric dipole moment & $\boldsymbol{m}$ & $\boldsymbol{p}_{\mathrm{SI}} /(4 \pi \epsilon)$ \\
Magnetic dipole moment & $\boldsymbol{m}$ & $\boldsymbol{m}_{\mathrm{SI}}[Z /(4 \pi)]$ \\
Electric-electric polarizability & $\boldsymbol{\alpha}_{E E}$ & $\boldsymbol{\alpha}_{E E}^{\mathrm{SI}} /(4 \pi \epsilon)$ \\
Magnetic-magnetic polarizability & $\boldsymbol{\alpha}_{H H}$ & $\boldsymbol{\alpha}_{H H}^{\mathrm{SI}} /(4 \pi)$ \\
Electric-magnetic polarizability & $\boldsymbol{\alpha}_{E H}$ & $\boldsymbol{\alpha}_{E H}^{\mathrm{SI}}[c /(4 \pi)]$ \\
Magnetic-electric polarizability & $\boldsymbol{\alpha}_{H E}$ & $\boldsymbol{\alpha}_{H E}^{\mathrm{SI}}[Z /(4 \pi)]$ \\
Electric-electric Green tensor & $\boldsymbol{G}_{E E}$ & $4 \pi \epsilon \boldsymbol{G}_{E E}^{\mathrm{SI}}$ \\
Magnetic-magnetic Green tensor & $\boldsymbol{G}_{H H}$ & $4 \pi \boldsymbol{G}_{H H}^{\mathrm{SI}}$ \\
Electric-magnetic Green tensor & $\boldsymbol{G}_{E H}$ & $4 \pi / Z \boldsymbol{G}_{E H}^{\mathrm{SI}}$ \\
Magnetic-electric Green tensor & $\boldsymbol{G}_{H E}$ & $4 \pi / c \boldsymbol{G}_{H E}^{\mathrm{SI}}$ \\
\hline \hline
\end{tabular}

to magnetoelectric coupling in stereodimers. This analysis is easily extended to magnetoelectric Yagi-Uda antennas, diffractive gratings of chiral building blocks, and magnetoinductive waveguides that may provide new ways to control the propagation and emission of light. ${ }^{57,75,76}$

\section{ACKNOWLEDGMENTS}

We thank Ad Lagendijk for stimulating and inspirative insights, as well as Dries van Oosten and Lutz Langguth for discussions. This work is part of the research program of the "Stichting voor Fundamenteel Onderzoek der Materie (FOM)," which is financially supported by the "Nederlandse Organisatie voor Wetenschappelijk Onderzoek (NWO)." A.F.K. acknowledges a NWO Vidi felloship. I.S. and A.F.K. both acknowledge support from "NanoNed", a nanotechnology program funded by the Dutch Ministry of Economic Affairs.

\section{APPENDIX: UNIT SYSTEM}

Throughout this paper, we used units that significantly simplify notation throughout, as they maximize the interchangeability of electric and magnetic fields. Conversion to SI units is summarized in Table I. For the conversion in Table I, we use $\epsilon$ for the host dielectric constant, $c$ for the velocity of light, and $Z$ for the impedance of the background medium. In this unit system, a plane wave has $|\boldsymbol{E}| /|\boldsymbol{H}|=1$ and intensity $I=|\boldsymbol{E}|^{2} /(2 Z)$, since the Poynting vector is $\boldsymbol{S}=1 /(2 Z) \operatorname{Re}\left(\boldsymbol{E}^{*} \times \boldsymbol{H}\right)$. In these units, the cycle-averaged work done by an electric field $\boldsymbol{E}$ to drive an oscillating $\boldsymbol{p}$ equals $W=2 \pi k / Z \operatorname{Im}(\boldsymbol{E} \cdot \boldsymbol{p})$. The magnetic counterpart is $W=2 \pi k / Z \operatorname{Im}(\boldsymbol{H} \cdot \boldsymbol{m})$ *i.sersic@amolf.nl; http://www.amolf.nl/research/resonant-nanophot onics/

${ }^{\dagger}$ Present address: Fritz-Haber-Institut der Max-Planck-Gesellschaft, Arnimallee 14, D-14195 Berlin, Germany.

${ }^{1}$ V. G. Veselago, Sov. Phys. Usp. 10, 509-514 (1968).

${ }^{2}$ J. B. Pendry, Phys. Rev. Lett. 85, 3966 (2000).

${ }^{3}$ J. B. Pendry, Physics World 14, 47 (2001); C. M. Soukoulis, S. Linden, and M. Wegener, Science 315, 47 (2007); V. M. Shalaev, Nat. Photon. 1, 41 (2007).

${ }^{4}$ U. Leonhardt, Science 312, 1777 (2006); J. B. Pendry, D. Schurig, and D. R. Smith, ibid. 312, 1780 (2006).

${ }^{5}$ D. R. Smith, W. J. Padilla, D. C. Vier, S. C. Nemat-Nasser, and S. Schultz, Phys. Rev. Lett. 84, 4184 (2000); W. J. Padilla, A. J. Taylor, C. Highstrete, M. Lee, and R. D. Averitt, ibid. 96, 107401 (2006); S. Linden, C. Enkrich, M. Wegener, J. Zhou, T. Koschny, and C. M. Soukoulis, Science 306, 1351 (2004).

${ }^{6}$ C. Enkrich, M. Wegener, S. Linden, S. Burger, L. Zschiedrich, F. Schmidt, J. F. Zhou, T. Koschny, and C. M. Soukoulis, Phys. Rev. Lett. 95, 203901 (2005).

${ }^{7}$ C. Rockstuhl, T. Zentgraf, H. Guo, N. Liu, C. Etrich, I. Loa, K. Syassen, J. Kuhl, F. Lederer, and H. Giessen, Appl. Phys. B 84, 219 (2006).

${ }^{8}$ M. W. Klein, C. Enkrich, M. Wegener, C. M. Soukoulis, and S. Linden, Opt. Lett. 31, 1259 (2006).

${ }^{9}$ I. Sersic, M. Frimmer, and A. F. Koenderink, Phys. Rev. Lett. 103, 213902 (2009).

${ }^{10}$ B. Lahiri, S. G. McMeekin, A. Z. Khokhar, R. M. De La Rue, and N. P. Johnson, Opt. Express 18, 3210 (2010).
${ }^{11}$ V. M. Shalaev, W. Cai, U. K. Chettiar, H.-K. Yuan, A. K. Sarychev, V. P. Drachev, and A. V. Kildishev, Opt. Lett. 30, 3356(2005).

${ }^{12}$ G. Dolling, C. Enkrich, M. Wegener, J. F. Zhou, C. M. Soukoulis, and S. Linden, Opt. Lett. 30, 23 (2005).

${ }^{13}$ G. Dolling, C. Enkrich, M. Wegener, C. M. Soukoulis, and S. Linden, Opt. Lett. 31, 12 (2006).

${ }^{14}$ G. Dolling, C. Enkrich, M. Wegener, C. M. Soukoulis, and S. Linden, Science 312, 892 (2007).

${ }^{15}$ J. Valentine, S. Zhang, T. Zentgraf, E. Ulin-Avila, D. A. Genov, G. Bartal, and X. Zhang, Nature (London) 455, 376 (2008).

${ }^{16}$ S. P. Burgos, R. de Waele, A. Polman, and H. A. Atwater, Nat. Mater. 9, 407 (2010).

${ }^{17}$ M. Husnik, M. W. Klein, N. Feth, M. König, J. Niegemann, K. Busch, S. Linden, and M. Wegener, Nat. Photon. 2, 614 (2008).

${ }^{18}$ C. Rockstuhl, F. Lederer, C. Etrich, T. Zentgraf, J. Kuhl, and H. Giessen, Opt. Express 14, 8827 (2006).

${ }^{19}$ T. D. Corrigan, P. W. Kolb, A. B. Sushkov, H. D. Drew, D. C. Schmadel, and R. J. Phaneuf, Opt. Express 16, 19850 (2008).

${ }^{20}$ A. Pors, M. Willatzen, O. Albrektsen, and S. I. Bozhevolnyi, J. Opt. Soc. Am. B 27, 1680 (2010).

${ }^{21}$ E. Prodan, C. Radloff, N. J. Halas, and P. Nordlander, Science 302, 419 (2003).

${ }^{22}$ P. Banzer, U. Peschel, S. Quabis, and G. Leuchs, Opt. Express 18, 10905 (2010).

${ }^{23}$ M. Burresi, D. van Oosten, T. Kampfrath, H. Schoenmaker, R. Heideman, A. Leinse, and L. Kuipers, Science 326, 550 (2009); M. Burresi, T. Kampfrath, D. van Oosten, J. C. Prangsma, B. S. Song, S. Noda, and L. Kuipers, Phys. Rev. Lett. 105, 123901 (2010). 
${ }^{24}$ N. Feth, M. König, M. Husnik, K. Stannigel, J. Niegemann, K. Busch, M. Wegener, and S. Linden, Opt. Express 18, 6545 (2010).

${ }^{25}$ M. Decker, S. Burger, S. Linden, and M. Wegener, Phys. Rev. B 80, 193102 (2009).

${ }^{26}$ J. K. Gansel, M. Thiel, M. S. Rill, M. Decker, K. Bade, V. Saile, G. von Freymann, S. Linden, and M. Wegener, Science 325, 1513 (2009).

${ }^{27}$ E. Plum, J. Zhou, J. Dong, V. A. Fedotov, T. Koschny, C. M. Soukoulis, and N. I. Zheludev, Phys. Rev. B 79, 035407 (2009).

${ }^{28}$ E. Plum, X.-X. Liu, V. A. Fedotov, Y. Chen, D. P. Tsai, and N. I. Zheludev, Phys. Rev. Lett. 102, 113902 (2009).

${ }^{29}$ S. Zhang, Y.-S. Park, J. Li, X. Lu, W. Zhang, and X. Zhang, Phys. Rev. Lett. 102, 023901 (2009).

${ }^{30}$ B. Wang, J. Zhou, T. Koschny, M. Kafesaki, and C. M. Soukoulis, J. Opt. A 11, 114003 (2009).

${ }^{31}$ E. Plum, V. A. Fedotov, and N. I. Zheludev, J. Opt. A 11, 074009 (2009).

${ }^{32}$ M. Decker, M. W. Klein, M. Wegener, and S. Linden, Opt. Lett. 32, 856 (2007).

${ }^{33}$ M. Decker, R. Zhao, C. M. Soukoulis, S. Linden, and M. Wegener, Opt. Lett. 35, 1593 (2010).

${ }^{34}$ N. Liu, H. Liu, S. Zhu, and H. Giessen, Nat. Photon. 3, 157 (2009).

${ }^{35}$ S. A. Tretyakov, A. H. Sihvola, A. A. Sochava, and C. R. Simovski, J. Electromagn. Waves Appl. 12, 481 (1998).

${ }^{36}$ E. Shamonina, V. A. Kalinin, K. H. Ringhofer, and L. Solymar, J. Appl. Phys. 92, 6252 (2002).

${ }^{37}$ M. G. Silveirinha, Phys. Rev. B 76, 245117 (2007).

${ }^{38}$ M. Gorkunov, M. Lapine, E. Shamonina, and K. H. Ringhofer, Eur. Phys. J. B 28, 262 (2002).

${ }^{39}$ R. Marqués, F. Medina, and R. Rafii-El-Idrissi, Phys. Rev. B 65, 144440 (2002).

${ }^{40}$ J. D. Baena, L. Jelinek, R. Marqués, and M. Silveirinha, Phys. Rev. A 78, 013842 (2008).

${ }^{41}$ A. N. Serdyukov, I. V. Semchenko, S. A. Tretyakov, and A. Sihvola, Electromagnetics of Bi-anisotropic Materials: Theory and Applications (Gordon and Breach, Amsterdam, 2001).

${ }^{42}$ H. Guo, N. Liu, L. Fu, T. P. Meyrath, T. Zentgraf, H. Schweizer, and H. Giessen, Opt. Express 15, 12095 (2007).

${ }^{43}$ J. Petschulat, J. Yang, C. Menzel, C. Rockstuhl, A. Chipouline, P. Lalanne, A. Tennermann, F. Lederer, and T. Pertsch, Opt. Express 18, 14454 (2010).

${ }^{44}$ H. Liu, J. X. Cao, S. N. Zhu, N. Liu, R. Ameling, and H. Giessen, Phys. Rev. B 81, 241403(R) (2010).

${ }^{45}$ C. Rockstuhl, T. Zentgraf, E. Pshenay-Severin, J. Petschulat, A. Chipouline, J. Kuhl, T. Pertsch, H. Giessen, and F. Lederer, Opt. Express 15, 8871 (2007).

${ }^{46}$ J. Zhou, Th. Koschny, and C. M. Soukoulis, Opt. Express 15, 17881 (2007).

${ }^{47}$ A. Lagendijk and B. A. van Tiggelen, Phys. Rep. 270, 143 (1996).

${ }^{48} \mathrm{P}$. de Vries, D. V. van Coevorden, and A. Lagendijk, Rev. Mod. Phys. 70, 2 (1998).

${ }^{49}$ F. J. García de Abajo, Rev. Mod. Phys. 79, 1267 (2007).

${ }^{50}$ P. A. Belov and C. R. Simovski, Phys. Rev. E 72, 026615 (2005).
${ }^{51} \mathrm{~J}$. Li and J. B. Pendry, e-print arXiv:cond-mat/0701332.

${ }^{52}$ L. D. Landau and E. M. Lifshitz, Electrodynamics of Continuous Media (Pergamon, Oxford, 1960).

${ }^{53}$ I. V. Lindell, A. H. Sihvola, S. A. Tretyakov, and A. J. Viitanen, Electromagnetic Waves in Chiral and Bi Isotropic Media (Artech House, Norwood, MA, 1994).

${ }^{54}$ P. A. Belov, S. I. Maslovski, K. R. Simovski, and S. A. Tretyakov, Tech. Phys. Lett. 29, 718 (2003) (translated from Russian).

${ }^{55}$ R. Merlin, Proc. Natl. Acad. Sci. USA 106, 1693 (2009).

${ }^{56}$ W. H. Weber and G. W. Ford, Phys. Rev. B 70, 125429 (2004).

${ }^{57}$ A. F. Koenderink and A. Polman, Phys. Rev. B 74, 033402(2006).

${ }^{58}$ J. Garcìa-Garcìa, F. Martìn, J. D. Baena, R. Marqès, and L. Jelinek, J. Appl. Phys. 98, 033103 (2005).

${ }^{59}$ C. F. Bohren and D. R. Huffman, Absorption and Scattering of Light by Small Particles (Wiley, New York, 1983).

${ }^{60}$ N. Katsarakis, T. Koschny, M. Kafesaki, E. N. Economou, and C. M. Soukoulis, Appl. Phys. Lett. 84, 2943 (2004).

${ }^{61}$ Note that the above equation should strictly be written with $\boldsymbol{\alpha}$ replaced by the $t$-matrix, which is directly proportional to the dynamic polarizability $\boldsymbol{\alpha}$ for point scatterers. ${ }^{47}$

${ }^{62}$ J. E. Sipe and J. Van Kranendonk, Phys. Rev. A 9, 1806 (1974).

${ }^{63}$ H. C. van de Hulst, Light Scattering by Small Particles (Dover, New York, 1981).

${ }^{64}$ M. Meier and A. Wokaun, Opt. Lett. 8, 581 (1983); K. T. Carron, W. Fluhr, A. Wokaun, and H. W. Lehmann, J. Opt. Soc. Am. B 3, 420 (1986); K. L. Kelly, E. Coronado, L. L. Zhao, and G. C. Schatz, J. Phys. Chem. B 107, 668 (2003); A. Wokaun, J. P. Gordon, and P. F. Liao, Phys. Rev. Lett. 48, 1574 (1982).

${ }^{65}$ A. Alù and N. Engheta, J. Nanophot. 4, 041590 (2010).

${ }^{66}$ M. Kerker, D. S. Wang, and C. L. Gilles, J. Opt. Soc. Am. 73, 765 (1983).

${ }^{67}$ The fact that $\boldsymbol{\alpha}_{\mathrm{SRR}}^{\text {static }}$ is not invertible is easily overcome by noting that the radiation damping theorem should be applied only to its nontrivial subspace, e.g., by replacing the zeros on the diagonal with infinitesimals.

${ }^{68}$ M. G. Silveirinha, Phys. Rev. B 82, 085101 (2010).

${ }^{69}$ R. Zhao, J. Zhou, Th. Koschny, E. N. Economou, and C. M. Soukoulis, Phys. Rev. Lett. 103, 103602 (2009); M. G. Silveirinha and S. I. Maslovski, ibid. 105, 189301 (2010); R. Zhao, J. Zhou, Th. Koschny, E. N. Economou, and C. M. Soukoulis, ibid. 105, 189302 (2010).

${ }^{70}$ P. B. Johnson and R. W. Christy, Phys. Rev. B 6, 4370 (1972).

${ }^{71}$ I. Sersic, A. Opheij, and A. F. Koenderink (unpublished).

${ }^{72}$ J. D. Jackson, Classical Electrodynamics, 3rd ed. (Wiley, New York, 1999).

${ }^{73}$ Note that the diagonal elements of the electrodynamic polarizability do contain contributions due to off-diagonal elements in the electrostatic tensor. The radiation damping correction in Eq. (22) mixes $\eta_{C}$ onto the diagonal.

${ }^{74}$ C. Menzel, T. Paul, C. Rockstuhl, T. Pertsch, S. Tretyakov, and F. Lederer, Phys. Rev. B 81, 035320 (2010).

${ }^{75}$ A. F. Koenderink, Nano Lett. 9, 4228 (2009).

${ }^{76}$ H. Liu, D. A. Genov, D. M. Wu, Y. M. Liu, J. M. Steele, C. Sun, S. N. Zhu, and X. Zhang, Phys. Rev. Lett. 97, 243902 (2006). 